\title{
Delayed pericardial effusion with tamponade after cardiac surgery
}

\author{
R. A. P. Scott and C. E. Drew \\ From the Thoracic Unit, Westminster Hospital, Horseferry Road, London
}

Seven cases of delayed pericardial effusion with tamponade in children have been reported previously, all following the Waterston operation. Three cases are now described in which delayed pericardial effusion developed three weeks after operation, following procedures other than the Waterston operation. Two of our cases, and two of the reported cases, died because the possibility of delayed pericardial effusion was not recognized. The previous reported cases are compared, and the possible aetiology is discussed.

Pericardial effusion producing a clinical picture similar to that of heart failure is well recognized as an early complication after open heart surgery for congenital heart disease. It had not been recorded as a late complication until 1969 when Somerville et al., reported 2 cases. Radley Smith, GonzalezLavin, and Somerville in 1970 reported 5 cases of delayed pericardial effusion, all after the Waterston operation (aorto-pulmonary anastomosis). Two patients died because the condition was not recognized.

Three cases of delayed pericardial effusion after other cardiac procedures are presented. Two patients died. In one the diagnosis was not made and in the other it was recognized too late.

\section{Case reports}

\section{Case I}

M.W. (male). Date of birth I8 January 1955. Operation at the age of 5 years for total anomalous pulmonary venous connexion to the coronary sinus. Pericardial effusion with tamponade developed during the fourth postoperative week.

History Full-term normal delivery weighing $3.1 \mathrm{~kg}$. The child presented in infancy as a feeding problem, with failure to thrive and peripheral cyanosis. Admitted in February 1966 at the age of I year, weighing $5.4 \mathrm{~kg}$.

Investigations Cardiac catheterization was performed, and the diagnosis of total anomalous pulmonary venous connexion to the coronary sinus was made. As the child's subsequent progress was satisfactory, operation was deferred until 1960 , by which time the child weighed $13.6 \mathrm{~kg}$.

Received 29 June I973.
Operation 9 May 1960 Through a median sternotomy, quadruple cannulation with cooling to $15^{\circ} \mathrm{C}$ (nasopharyngeal) was carried out, and a complete correction of the lesion was made during circulatory arrest.

Postoperative course The child recovered well, but required digitalis for mild congestive cardiac failure. By the 4th postoperative day, there were no signs of failure. On the 13th postoperative day the child was allowed out of bed and then transferred to another hospital for convalescence.

Three weeks after operation, examination revealed no heart failure and the child was active and well. A few days later, the child started to vomit, the liver was enlarged, and there was mild cyanosis. A clinical diagnosis of recurrent congestive cardiac failure was made. Four weeks after operation pulsus paradoxus was noted, the jugular venous pressure was raised, and the liver edge was 4 finger breadths below the costal margin. A chest radiograph showed a considerable increase in the cardiac silhouette. By now the child was extremely ill and the diagnosis of pericardial effusion with tamponade was made.

Operation 13 June 1960 The lower end of the sternotomy wound was reopened and $800 \mathrm{ml}$ straw-coloured fluid were released. There was no evidence of infection and the heart was small.

Postoperative course At the time of operation, the child was in gross electrolyte imbalance ( $\mathrm{Na} \mathrm{125;} \mathrm{Cl} 85$; $\mathrm{K}$ 3.2; total protein 6 g.). Despite an initial improvement, it proved impossible to correct the electrolyte abnormalities quickly enough and the child died 12 hours after operation.

Necropsy The pericardial effusion had not recurred and the original lesion had been completely corrected.

Investigation of fluid removed at operation Total protein $4.5 \mathrm{~g} / 100 \mathrm{ml}$. No growth or culture. No organisms seen. No growth at 6 weeks for acid-fast bacilli. 


\section{Case 2}

J.A. (male). Date of birth 5 December 1953. Operation at the age of ro years for persistent ostium secundum. Pericardial effusion with tamponade occurred 3 weeks after operation.

History Full-term normal delivery at home. Birthweight $2.9 \mathrm{~kg}$. Not cyanosed. Normal milestones. A heart murmur was first noticed when he was admitted to hospital at the age of 8 years for gastroenteritis. The mother had noticed that he became cyanosed on running.

In 1963 at the age of Io years the child was referred for cardiac investigation.

Investigation Cardiac catheterization confirmed the clinical diagnosis of persistent ostium secondum.

Operation 17 June 1963 Surface cooling to $25^{\circ} \mathrm{C}$ (oesophageal) was carried out. The defect was closed by direct suture with venous inflow occlusion. This technique was used because the child's blood group was the rare $A_{2} B$ with anti $A_{1}$, and sufficient blood could not be obtained for a bypass technique.

Postoperative course The child made an uneventful recovery. He was transferred on the 13th postoperative day to another hospital for convalescence. On the rgth postoperative day he vomited once and 23 days after operation he complained of pain in the epigastrium and vomited again. Examination showed engorged neck veins with a prominent $A$ wave. The liver was enlarged 2 fingers breadths below the costal margin. Heart sounds were distant and the apex beat impalpable. A diagnosis of pericardial effusion with tamponade was made.

Operation Io July 1963 Through a vertical incision at the xiphoid process (Cassel and Callum, 1967) $600 \mathrm{ml}$ pink, clear fluid was released, and the pericardial space drained.

Postoperative course A further $530 \mathrm{ml}$ fluid drained over the following 3 days. He made a good recovery and was discharged on 2I July 1963. Twelve days later he was seen in the outpatients department and was readmitted because of recurrence of the pericardial effusion. Over the next 2 months he required repeated aspirations for recurring effusions. Drainage of the pericardial space was performed for a second time on 12 September 1963 when $1,500 \mathrm{ml}$ clear straw-coloured fluid were released.

At the time of the last operation steroids were administered and the effusion did not reaccumulate. Four years later in 1967 he was well with no murmur and a normal chest radiograph.

Investigation of fluid removed at operation Lymphocytes roo per cent. No organisms seen. No growth on culture. Culture for acid-fast bacilli negative.

\section{Case 3}

N.E. (male). Date of birth 26 January 1959. Operation at the age of II years for persistent ostium primum defect with mitral regurgitation. Pericardial effusion with tamponade developed 4 weeks after operation.
History Full-term normal delivery, with normal milestones. A heart murmur was first heard at a routine school medical examination when the child was 8 years old.

Investigation At cardiac catheterization, a clinical diagnosis of ostium primum defect with mitral regurgitation was confirmed.

Operation 30 April 1970 Through a median sternotomy, quadruple cannulation with cooling to $15^{\circ} \mathrm{C}$ (nasopharyngeal) was carried out. The cleft in the anterior cusp of the mitral valve was repaired and the atrial septal defect closed by direct suture.

Postoperative course The child made a good recovery, but required epicardial pacing for heart block for the first 4 postoperative days, after which there was reversion to normal rhythm. Chest radiograph on the 4th postoperative day was satisfactory.

On the 7th postoperative day, the child was transferred to another hospital for convalescence.

At that time he was noted to have a regular pulse of 95, a raised jugular venous pressure $5 \mathrm{~cm}$ above the clavicle, and an enlarged liver 2 finger breadths below the costal margin. He was found to be anaemic and was given oral iron. Two weeks later he was much improved and fully mobile. He was discharged home three weeks after his operation with a haemoglobin of $9.5 \mathrm{~g} / 100 \mathrm{ml}$.

Ten days after being discharged (4 weeks after operation) he was admitted as an emergency to another hospital with cyanosis, vomiting, an enlarged liver 5 finger breadths below the costal margin, and engorged neck veins. The apex beat was not palpable. The child was treated for congestive cardiac failure, but died a few hours after admission.

Necropsy There was a large clear pericardial effusion, the heart was small, the mitral valve well repaired, and the septal defect closed. Histology showed normal myocardium, with some patchy areas of inflammatory response to the suture material.

\section{Discussion}

The three cases presented show two important differences, when compared with those reported by Somerville et al. and Radley-Smith et al. The first is the later onset. The cases reported by these authors occurred within the first 3 postoperative weeks, 2 before the 14 th postoperative day. It was suggested that this complication might be prevented by keeping the patients in hospital for at least 14 days after the operation. All our cases presented after the 3rd postoperative week, and one 5 weeks after the operation. Because of their uncomplicated immediate postoperative course these patients were transferred or discharged relatively early. It was not until 3 to 5 weeks after operation that deterioration occurred.

The second difference is that all of the cases of Somerville and Radley-Smith were associated with a 
Waterston anastomosis; indeed these authors speculated as to whether some special feature of the operation was responsible. None of our cases had a Waterston anastomosis, the only common factor being that they all underwent closure of an atrial septal defect, with or without associated defects.

The incidence of delayed pericardial effusion with tamponade in our experience is $I$ in 400 open heart operations for congenital heart disease, and would be considerably lower if all cases involving pericardiotomy were considered.

The cause of delayed pericardial effusion is unknown. It appears to be unrelated to any failure in operative technique, since the 2 cases that came to necropsy had small hearts in a greatly distended pericardial sac and the result of the surgical operation was satisfactory.

In one patient the effusion contained roo per cent lymphocytes, suggesting a viral or autoimmune reaction. The delay in the formation of the effusion could be related either to the time taken for the pericardium to reform as an epithelial lined sac or, if an autoimmune aetiology is postulated, to the time taken for the autoimmune process to develop. Unfortunately we have no antibody studies on these cases.

Radley-Smith et al. (1970) suggested that the presence of pulmonary atresia, or the relatively small size of their pericardial incision might be the cause of the effusion. None of our cases had pulmonary atresia and all our cases had the pericardial sac opened widely and subsequently loosely repaired. It is interesting that none of our cases had any adhesions between the heart and pericardium nor a pyrexia during their period of deterioration (Engelman et al., 1970; Prewitt et al., 1968).

Once the complication has been recognized, drainage by incision at the xiphisternum is the most effective way of treating the immediate problem. The use of steroids appears to prevent recurrence of the effusion.

\section{References}

Cassel, P., and Callum, P. (1967). Management of cardiac tamponade. British fournal of Surgery, 54, 620.

Engelman, R. M., Spencer, F. C., Reed, G. E., and Tice, D. A. (1970). Cardiac tamponade following open-heart surgery. Circulation, 4I-42, Suppl. 2. I65.

Prewitt, T. A., Rackley, C. E., Wilcox, B. R., Scatliff, J. H., and Young, D. T. (I968). Cardiac tamponade as a late complication of open-heart surgery. American Heart fournal, 76, 139.

Radley-Smith, R., Gonzalez-Lavin, L., and Somerville, J. (1970). Pericardial effusion with tamponade following anastomosis of the ascending aorta to the right pulmonary artery (Waterston's operation). Fournal of Thoracic and Cardiovascular Surgery, 60, 565 .

Somerville, J., Yacoub, M., Ross, D. N., and Ross, K. (1969). Aorta to right pulmonary artery anastomosis (Waterston's operation) for cyanotic heart disease. Circulation, 39, 593.

Requests for reprints to R. A. P. Scott, Esq., F.R.C.S., 4 Guildhouse Street, London S.W.I. 